\title{
LETTER
}

Myelodysplastic syndrome

\section{Activating somatic and germline TERT promoter variants in myeloid malignancies}

\author{
Valeria Nofrini ${ }^{1}$ - Caterina Matteucci ${ }^{1}$. Fabrizia Pellanera ${ }^{1}$ Paolo Gorello ${ }^{1}$ - Danika Di Giacomo ${ }^{1}$. \\ Anair Graciela Lema Fernandez ${ }^{1}$. Carlotta Nardelli ${ }^{1}$. Tamara lannotti ${ }^{1} \cdot$ Lucia Brandimarte $^{1} \cdot$ Silvia Arniani $^{1}$. \\ Martina Moretti ${ }^{1} \cdot$ Alessio Gili $^{2} \cdot$ Giovanni Roti $^{3} \cdot$ Valeria Di Battista $^{1} \cdot$ Simona Colla $^{4} \cdot$ Cristina Mecucci $^{1}$
}

Received: 23 December 2019 / Revised: 12 March 2020 / Accepted: 8 April 2020 / Published online: 4 May 2020

(c) The Author(s) 2020. This article is published with open access

\section{To the Editor:}

TERT gene is encoding for the telomerase enzyme catalytic subunit, which maintains genomic integrity through de novo synthesis of telomere repeats at chromosome ends. It is active in stem and germinal cells, thus sustaining physiological replication [1]. TERT is silenced in somatic cells where progressive telomere erosion, along with cell division, induces senescence and genetic alterations [1]. Congenital variations at the TERT coding sequence and of a number of genes involved in telomere biology are known in dyskeratosis congenita that is the prototype of telomere-related disorders, mainly affecting skin, lung, bone marrow (BM), and liver [2]. In cancer aberrant TERT expression contributes to immortalization through specific mechanisms inducing telomerase reactivation $[1,3]$. This may occur by means of both methylation and mutations at TERT promoter $\left(T E R T_{P}\right)$ [3]. In acute myeloid leukemia (AML), hypermethylation at THOR (TERT hypermethylated oncological region) was

These authors contributed equally: Valeria Nofrini, Caterina Matteucci

Supplementary information The online version of this article (https:// doi.org/10.1038/s41375-020-0837-6) contains supplementary material, which is available to authorized users.

Cristina Mecucci

cristina.mecucci@unipg.it

1 University of Perugia, Section of Hematology and Center for Hemato-Oncology Research (C.R.E.O.), Perugia, Italy

2 Public Health Section, Department of Experimental Medicine, University of Perugia, Perugia, Italy

3 Hematology and Bone Marrow Transplantation Unit, University of Parma, Parma, Italy

4 Department of Leukemia, The University of Texas MD Anderson Cancer Center, Houston, TX, USA frequently found [4]. In solid tumors, somatic C $>$ T hotspot transition at -124 and -146 nucleotides from the TERT ATG start site, and other rare mutations $(-57 \mathrm{~A}>\mathrm{C}$; $-124 /-125 \mathrm{CC}>\mathrm{TT}$; $-138 /-139 \mathrm{CC}>\mathrm{TT})$, are functionally activating by creating de novo binding sites for Etwenty-six (ETS) transcription factors [1]. Information about mutations in hematopoietic malignancies is scarce. To the best of our knowledge, $T E R T_{P}$ hotspot mutations have been described only in mantle cell lymphomas [5].

Here, we investigated $T E R T_{P}$ variants in a large series of myelodysplastic syndromes (MDS) and MDS/myeloproliferative neoplasms (MDS/MPN). Biological samples [cytogenetic preparations, genomic DNA, frozen and fresh $\mathrm{BM}$, peripheral blood (PB) cells and nail cuttings] were obtained from patients referred to the Hematology Unit at the University of Perugia between 1995 and 2019. The study was conducted according to Helsinki declaration and approved by the Institutional Bioethics Committee (University of Perugia Protocol No. 2017-19R). Written informed consent was obtained from all patients and controls. New and rare (i.e., minor allele frequency <0.01) $T_{E R T_{P}}$ variants were analyzed in silico through JASPAR Database and in vitro using Luciferase Reporter assay (Supplementary Table 1). TERT $T_{P}$-positive cases were further screened by Sanger Sequencing for the rs2853669 T>C single-nucleotide polymorphism, since it was previously shown to modulate mutated $T E R T_{P}$ in cis [6]. In addition, 30 myeloid leukemogenic genes and 35 telomere-related genes were investigated by next generation sequencing (NGS) using, respectively, the commercial Myeloid solution $^{\mathrm{TM}}$ and a Custom Hereditary Hematological Disorders gene panel provided by SOPHiA Genetics (Saint Sulpice, Switzerland). Telomere length (TL) was measured by Quantitative-Fluorescence In Situ Hybridization (Q-FISH) and/or Quantitative PCR (qPCR). For additional details, see Supplementary Methods, available on the Leukemia website. 
We recruited $37 \mathrm{MDS} / \mathrm{MPN}$ and $350 \mathrm{MDS}$ (250 males, 137 females; median age 74, range 7-94). Supplementary Table 2 shows demographics, hematological and cytogenetic features of all cases.

Sanger sequencing revealed $T E R T_{P}$ variants in $6 / 387$ cases $(1.5 \%$, Table 1), including five MDS and one chronic myelomonocytic leukemia (CMML). TERT $P$ variants affected the hotspot nucleotide at -124 base pairs upstream to the TERT ATG start site in three cases. Two of them carried the c.1-124C $>\mathrm{T}$ hotspot, while the third one had a C $>$ A substitution (Table 1). Both of these variants were previously shown to significantly increase $T E R T_{P}$ activity [7]. Two more cases bore hitherto unknown variants, which were not found neither in our screening of PB from 200 healthy controls nor in dedicated databases, i.e., a c.1110_1-101dup and a germline c.1-71G>C (Fig. 1a, Supplementary Table 3). The last case of this series carried a germline c.1-78T>C rs1467435130 variant (Fig. 1a, Table 1).

Sequencing and in silico analysis (JASPAR Database) showed the $T E R T_{P}$ c.1-110_1-101dup produced duplication of binding sites for Sp1, a member of Specificity Protein/ Krüppel-Like Factor transcription factor family (Fig. 1a and Supplementary Table 4) that, similarly to ETS family, was associated with $T E R T_{P}$ activation [1]. A significantly increased binding of transcription factors belonging to the same families was also generated by the two germline variants (c.1-71G $>\mathrm{C}$ and c.1-78T $>\mathrm{C}$, Supplementary Table 4).

In two families with the same c.1-57A $>C$ variant and early onset melanoma, a pathogenetic role in cancer development was suggested for the germline $T E R T_{P}$ variant [1]. In the family bearing the c.1-78T $>C$ substitution, however, neither blood nor solid tumors emerged in six carriers identified across three generations (age range 18-73 years, mean age 47.5, median 52), suggesting that our variant "per $s e$ " is not promoting malignancy (Supplementary Fig. 1).

According to in silico analysis, the Luciferase Reporter assay showed that all three variants caused a significantly increased $T E R T_{P}$ activity by $1.3-1.7$ fold in HeLa cells (Fig. 1b). Notably, adding evidence for a cell contextdependent action [6], the rs2853669 polymorphic $\mathrm{C}$ allele had no effect against our c.1-110_1-101dup (Fig. 1b).

As recurrent $T E R T_{P}$ mutations have been previously found in individuals with telomerase deficiency due to pathogenetic variants at the coding sequence of TERT, TERC and PARN [8, 9], we tested these genes and extended our NGS analysis to 32 additional telomere-related genes. Results showed six heterozygous variants classified as benign, likely benign, or of uncertain significance, according to the American College of Medical Genetics criteria (Table 1, Supplementary Methods). Among them there were two synonymous (TERT, RIF), two missense (RECQL4, TERF2), one splice site (TERT) variant and one inframe duplication $(D K C l)$. In addition, we found a pathogenetic frameshift heterozygous variant at $R A D 50$ (Table 1). Although the significance of this observation remains to be clarified, deleterious $R A D 50$ variants in heterozygosity have never been reported in a telomere-related phenotype [10]. Based on all these results, we excluded a congenital defect of the telomerase underlying $T E R T_{P}$ variants in this series.

We further extended our investigations to dysplastic BM cells by both conventional cytogenetics and a myeloid NGS panel. Acquired cytogenetic aberrations and/or mutations were identified in all cases, suggesting cooperation between $\operatorname{TERT}_{P}$ and disease-related somatic hits (Table 1). Similarly, $T E R T_{P}$ mutations were previously reported in bladder cancer with FGFR3 mutations and in both thyroid cancer and melanoma with $B R A F$ mutations, favoring the hypothesis that telomerase reactivation supports the proliferation of oncogene-transformed cells [1]. However, since telomerase is constitutively active in hematopoietic stem cells, the significance of its activation in myeloid malignancies is less clear [1].

Predominant somatic events in our cases were loss of function mutations at one or more epigenetic genes, namely, TET2 and EZH2. Interestingly in murine embryonic stem cells, TET2 deficiency led to sub-telomeric hypermethylation and telomere shortening via a telomerase-independent effect [11]. In human glioma cell lines, EZH2 depletion reduced TERT level [12]. Its inhibition in a human osteosarcoma cell line decreased telomeric heterochromatic marks [13]. Furthermore, in human AML cell lines, DNA methyltransferases inhibition by 5-azacytidine induced DNA damage at telomeres, telomere shortening, and downregulation of TERT expression [14]. Altogether these data suggest a functional link between epigenetic genes and $T E R T_{P}$ variants, that in our series might have counterbalanced acquired telomere disturbances. Although this hypothesis remains to be proved, insights were generated from studies on TL. First, in three $T E R T_{P}$ cases positive for TET2 (and also for EZH2 in one out of three) the TL in PB was not reduced, compared with age- and sex-matched healthy controls (Fig. 1c). This was rather unexpected as telomere shortening is typically found in MDS [15]. Moreover, in intra-individual longitudinal studies TL in BM cells was almost stable over time (Fig. 1d) and it was similar or even slightly longer than that of $\mathrm{CD}^{+}$lymphocytes without epigenetic mutations (Fig. 1e, Supplementary Fig. 2). Notably, in MDS telomere attrition was shown to preferentially affect the myeloid versus the lymphoid compartment [16].

In conclusion, our study for the first time identified somatic and germline activating $T E R T_{P}$ variants in both 


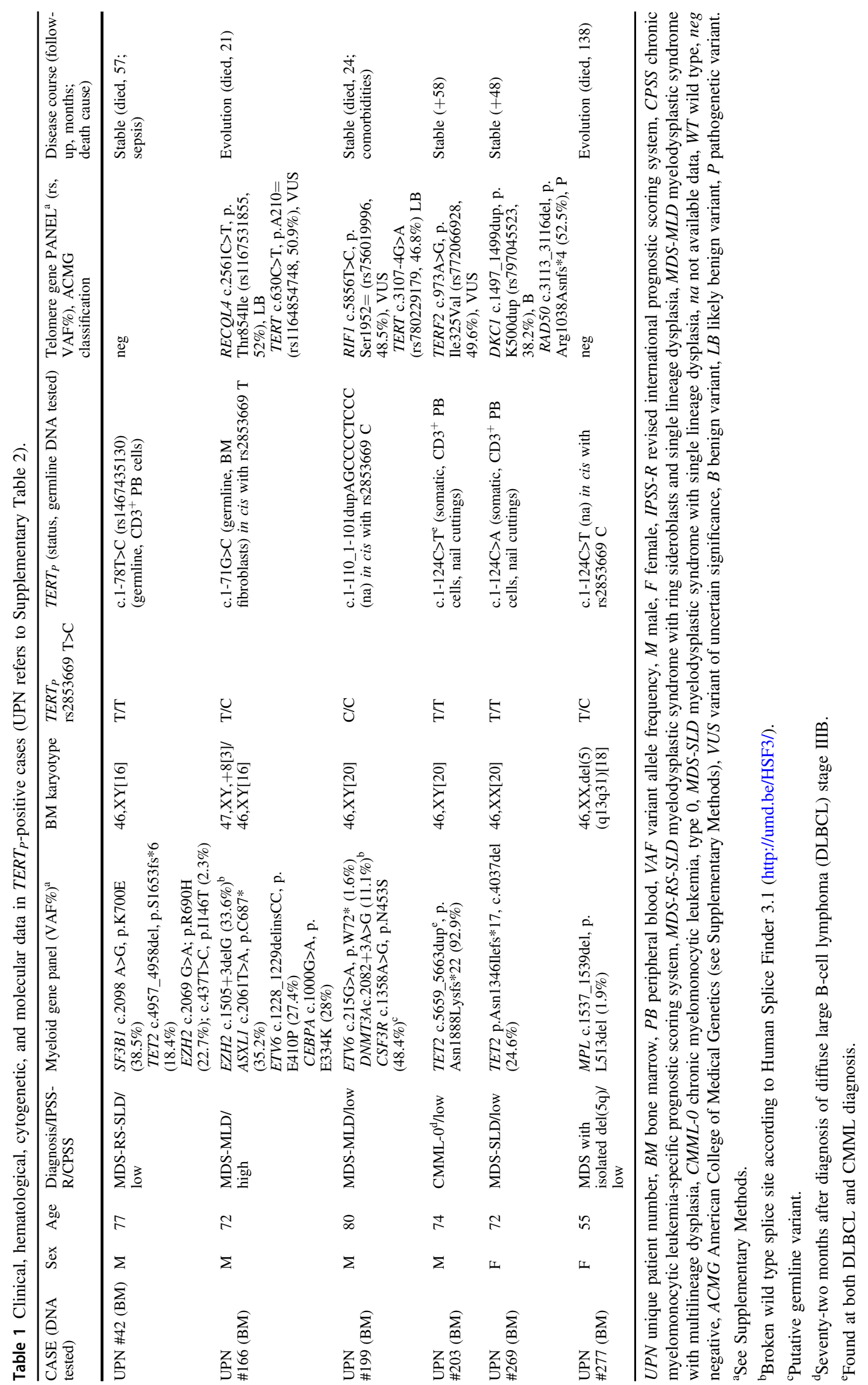


a

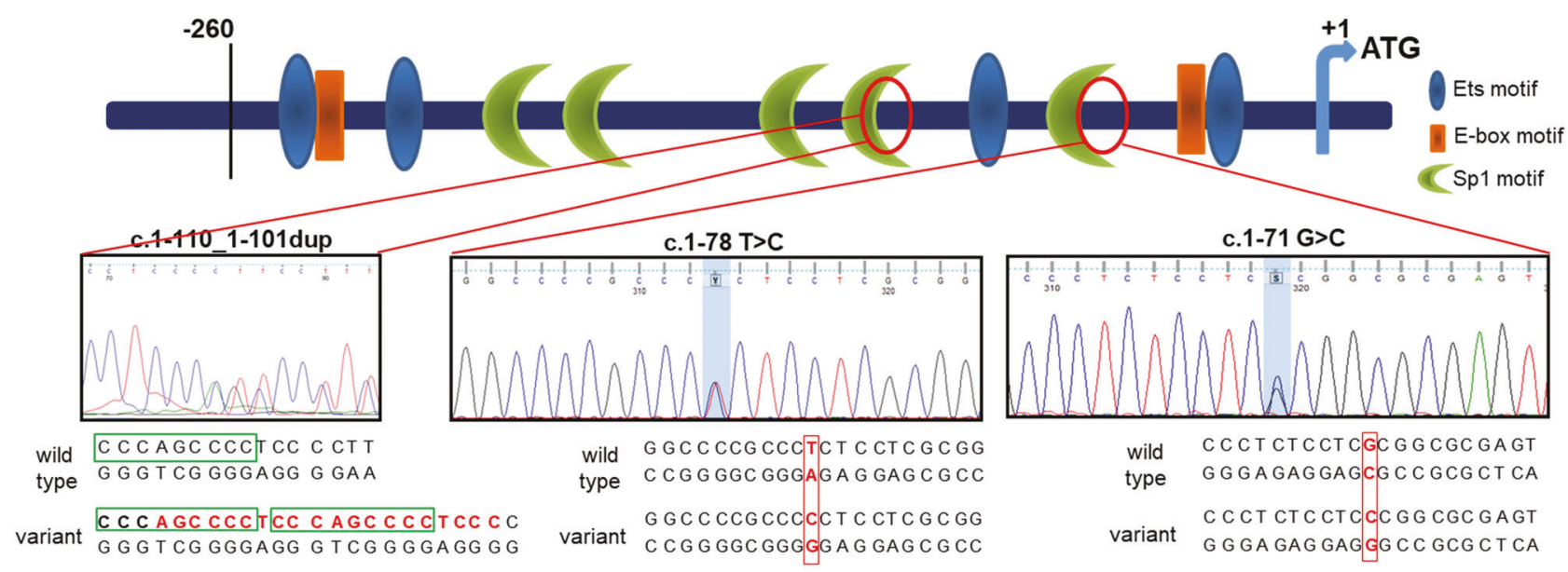

b

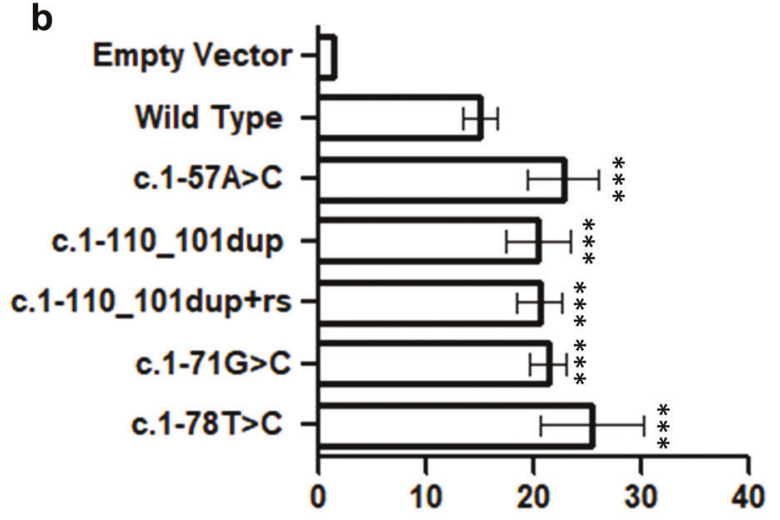

Relative Luciferase Activity

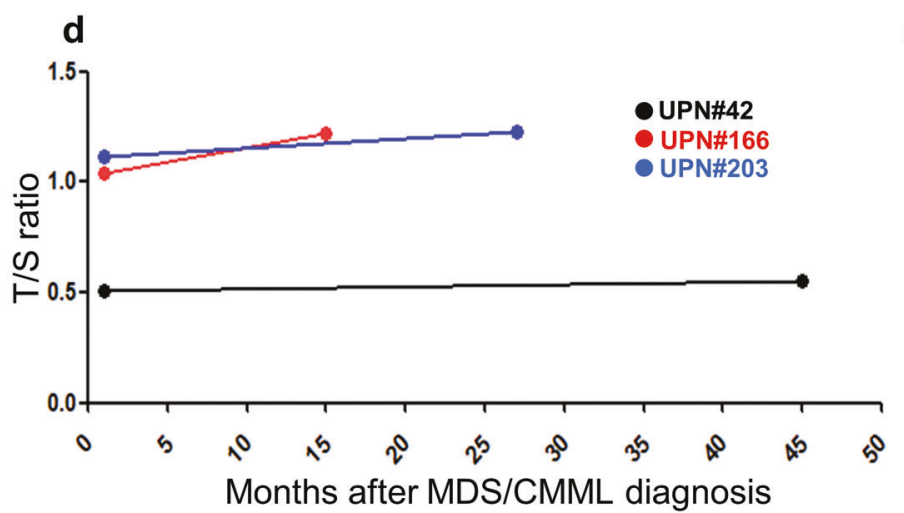

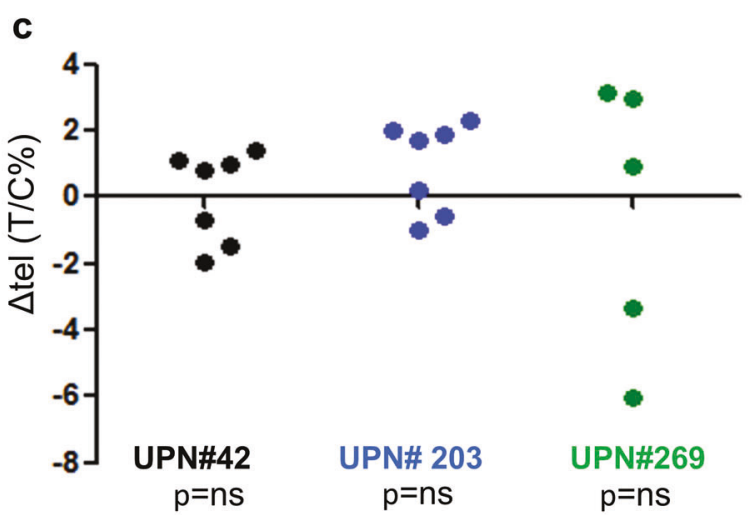

e

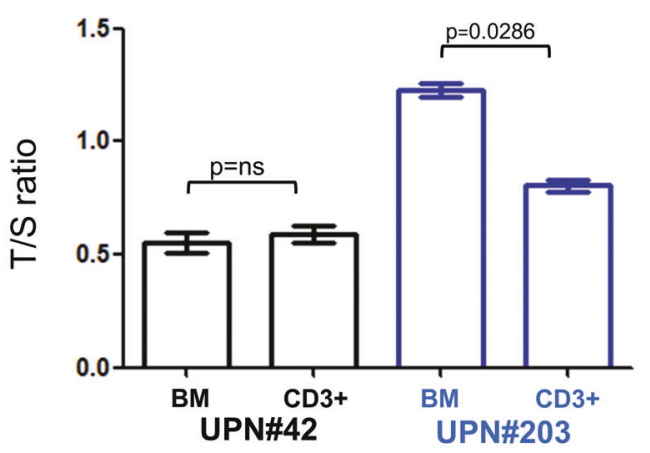

Fig. 1 New and rare $T E R T_{P}$ activating variants increased promoter activity and TL investigations in malignant BM and PB cells from TERT $_{P}$-positive cases did not reveal shortening as expected. a Schema of $T E R T_{P}$ with transcription factor binding sites (blue, orange, and green symbols) and new/rare TERT $T_{P}$ variants in myeloid malignancies (red circles). The c.1-110_1-101dup included duplication of an Sp1 binding site (green square), between nucleotides 1295095 and 1295102 (ENSG00000164362 GRCh38.p12). Adapted from Heidenreich and Kumar ${ }^{1}$ with permission. b Relative Luciferase activity in HeLa cell line; rs refers to rs2853669 T $>$ C. Data are shown as mean \pm SD in four independent experiments. The construct with the known activating c.1-57A $>\mathrm{C}$ was used as an internal positive control to validate data. $* * * p<0.001$ vs. wild type (two sample $t$-test with equal variances). c Inter-individual TL analysis by Q-FISH on PHA-stimulated PB metaphases. Unique patient number (UPN) refers to Supplementary Table 2. TL is expressed as T/C\%. Each dot represents the difference ( $\Delta \mathrm{TL}$ T/C\%) between mean TL in each patient and each age- and sex-matched healthy control (for UPN\#42 and \#203: 7 males, age range 71-83, mean 78.2, median 81, and for UPN\#269: 5 females, age range 64-75, mean 72, median 74). ns, not significant (one sample Student's $t$ test). d Intra-individual TL over time according to qPCR on BM DNA at diagnosis and during the disease course. TL is expressed as T/S ratio. e Intra-individual TL analysis by qPCR comparing BM DNA with PB CD3 ${ }^{+}$cells negative for somatic mutations. TL is expressed as T/S ratio. Data are shown as mean $\pm \mathrm{SD}$ in four independent experiments $(p<0.05$, Mann-Whitney $U$ test). 
MDS and CMML and provided insights on their close association with acquired mutations at epigenetic modulators. Inclusion of $T E R T_{P}$ screening in the diagnostic routine of MDS and MDS/MPN will be helpful to better assess the biological and clinical significance of $T E R T_{P}$ variants.

Acknowledgements The authors wish to thank Dr Geraldine Boyd for assistance in the preparation of the paper; Dr Pellegrino Musto for providing clinical-hematological findings in one case; Dr Sharon Savage and Dr Casey Dagnall for advices to setup qPCR for telomere length measurement. This work was supported by AIRC $5 \times 1000$, MYNERVA project, \#21267 (Myeloid Neoplasms Research Venture Airc, http://www.progettoagimm.it). GR was supported by AIRC Start-up Investigator Grant (No. 17107 G.R.).

Author contributions CMe conceived and supervised the study. VN, CMa, FP, TI, SA, and MM performed cytogenetics, mutational analysis, and cloning experiments. VN, PG, and DDG performed and analyzed telomere length experiments. AGLF, LB, and AG performed functional studies and analyzed results. GR and VDB collected clinical data and patient samples. SC revised the paper. $\mathrm{CMe}, \mathrm{VN}$, and $\mathrm{CMa}$ wrote the paper.

\section{Compliance with ethical standards}

Conflict of interest The authors declare that they have no conflict of interest.

Publisher's note Springer Nature remains neutral with regard to jurisdictional claims in published maps and institutional affiliations.

Open Access This article is licensed under a Creative Commons Attribution 4.0 International License, which permits use, sharing, adaptation, distribution and reproduction in any medium or format, as long as you give appropriate credit to the original author(s) and the source, provide a link to the Creative Commons license, and indicate if changes were made. The images or other third party material in this article are included in the article's Creative Commons license, unless indicated otherwise in a credit line to the material. If material is not included in the article's Creative Commons license and your intended use is not permitted by statutory regulation or exceeds the permitted use, you will need to obtain permission directly from the copyright holder. To view a copy of this license, visit http://creativecommons. org/licenses/by/4.0/.

\section{References}

1. Heidenreich B, Kumar R. TERT promoter mutations in telomere biology. Mutat Res. 2017;771:15-31.
2. Niewisch MR, Savage SA. An update on the biology and management of dyskeratosis congenita and related telomere biology disorders. Expert Rev Hematol. 2019;12:1037-52.

3. Barthel FP, Wei W, Tang M, Martinez-Ledesma E, Hu X, Amin $\mathrm{SB}$, et al. Systematic analysis of telomere length and somatic alterations in 31 cancer types. Nat Genet. 2017;49:349-57.

4. Lee DD, Leão R, Komosa M, Gallo M, Zhang CH, Lipman T, et al. DNA hypermethylation within TERT promoter upregulates TERT expression in cancer. J Clin Invest. 2019;129:223-9.

5. Panero J, Alves-Paiva RM, Roisman A, Santana-Lemos BA, Falcão RP, Oliveira G, et al. Acquired TERT promoter mutations stimulate TERT transcription in mantle cell lymphoma. Am J Hematol. 2016;91:481-5.

6. Ko E, Seo HW, Jung ES, Kim BH, Jung G. The TERT promoter SNP rs2853669 decreases E2F1 transcription factor binding and increases mortality and recurrence risks in liver cancer. Oncotarget. 2016;7:684-99.

7. Bell RJ, Rube HT, Kreig A, Mancini A, Fouse SD, Nagarajan RP, et al. Cancer. The transcription factor GABP selectively binds and activates the mutant TERT promoter in cancer. Science. 2015;348:1036-9.

8. Maryoung L, Yue Y, Young A, Newton CA, Barba C, van Oers NS, et al. Somatic mutations in telomerase promoter counterbalance germline loss-of-function mutations. J Clin Invest. 2017;127:982-6.

9. Gutierrez-Rodrigues F, Donaires FS, Pinto A, Vicente A, Dillon LW, Clé DV, et al. Pathogenic TERT promoter variants in telomere diseases. Genet Med. 2019;21:1594-602.

10. Taylor AMR, Rothblum-Oviatt C, Ellis NA, Hickson ID, Meyer $\mathrm{S}$, Crawford TO, et al. Chromosome instability syndromes. Nat Rev Dis Prim. 2019;5:1-19.

11. Yang J, Guo R, Wang H, Ye X, Zhou Z, Dan J, et al. Tet enzymes regulate telomere maintenance and chromosomal stability of mouse ESCs. Cell Rep. 2016;15:1809-21.

12. Ahmad F, Patrick S, Sheikh T, Sharma V, Pathak P, Malgulwar $\mathrm{PB}$, et al. Telomerase reverse transcriptase (TERT)-enhancer of zeste homolog 2 (EZH2) network regulates lipid metabolism and DNA damage responses in glioblastoma. $\mathrm{J}$ Neurochem. 2017;143:671-83.

13. Montero JJ, López-Silanes I, Megías D, Fraga FM, CastellsGarcía Á, Blasco MA. TERRA recruitment of polycomb to telomeres is essential for histone trymethylation marks at telomeric heterochromatin. Nat Commun. 2018;9:1548.

14. Zhang X, Li B, de Jonge N, Björkholm M, Xu D. The DNA methylation inhibitor induces telomere dysfunction and apoptosis of leukemia cells that is attenuated by telomerase over-expression. Oncotarget. 2015;6:4888-900.

15. Yang L, Mailloux A, Rollison DE, Painter JS, Maciejewski J, Paquette RL, et al. Naive T-cells in myelodysplastic syndrome display intrinsic human telomerase reverse transcriptase (hTERT) deficiency. Leukemia. 2013;27:897-906.

16. Rollison DE, Epling-Burnette PK, Park JY, Lee JH, Park H, Jonathan $\mathrm{K}$, et al. Telomere length in myelodysplastic syndromes. Leuk Lymphoma. 2011;52:1528-36. 